\title{
The functional form of nuclei decay in a thermal point-reactor within the particles' birth and death model
}

\author{
Maksim Kravchenko ${ }^{1, *}$, Eduard Rudak $^{1}$, Tamara Korbut ${ }^{1}$, Andrey Kuzmin ${ }^{1}$, and Andrey \\ Petrovski $^{1}$ \\ ${ }^{1}$ The State Scientific Institution «The Joint Institute for Power and Nuclear Research - Sosny» of the \\ National Academy of Sciences of Belarus, 220109 Minsk, Republic of Belarus
}

\begin{abstract}
The functional form of nuclei decay for a finite number of particles within sub-Poisson distribution terms and time scale discretisation were considered. The mathematical apparatus for particles' birth and death model with respect to the theory of a nuclear thermal point-reactor was made. During research the fundamental curves, the exponential dependence of neutron generation lifetime and state decay possibility were obtained for a thermal reactor breeding medium. Main parameter of a breeding (neutron multiplication factor $K$ ) was obtained through mathematical functions considered.
\end{abstract}

\section{Introduction}

For many processes in many fields of science the problem of mean value estimation is solved by an adequate probabilistic process. After choosing this probabilistic process the instantaneous intensities are build. The mathematical expectations of random variables and their variances can be estimated with analytical functions from transient intensities. This idea is also suitable for describing a thermal nuclear reactor.

Historically the physics of nuclear reactor was built on the basis of data gained from numerous experiments. The idea of applying general mathematical apparatus of probability theory for describing nuclear reactor processes seemed to be nonsense. On the other hand, authors made a successful attempt to create a mathematical apparatus for a simple point reactor within the birth and death model framework using the Kolmogorov equations [1].

In [2] was described a subcritical thermal reactor with inner neutron source in the form of delayed neutrons emitter nuclei. The same idea was introduced in [3], but never taken into account as was blamed to be physically incorrect. Described in [2] subcritical reactor was assumed to be an analog of Accelerator Driven Subcritical systems (ADS-systems), as one described in [4]. But thermal reactors should also be considered to be stable systems as they operate in a subcritical on prompt neutrons state with $\rho=-\beta$ ( $\beta$ is total delayed neutrons output). The birth and death model gives the explanation of such a view.

* Corresponding author: max.slevin.grey@gmail.com 
In current work the definitive mathematical apparatus of birth and death model is considered. Starting from the basics of neutron evolution in breeding medium the total neutron multiplication factor was obtained in analytical form for a thermal point-reactor.

\section{Theoretical basics}

In [2] the first basics of implementing birth and death model to the reactor physics were made. It was shown, that for processes in reactor breeding medium can be described with probabilistic mathematical apparatus, where the mathematical expectation of particles number can be found. That assumption is suitable due to the fact of discrete form of nuclei transformation processes. The interaction of neutron with a breeding medium is connected with calculation of specific for birth and death model integral.

$$
I(t)=\exp \left\{\int_{0}^{t}[\lambda(s)-\mu(s)] d s\right\} .
$$

The function under integral in equation (1) is the difference between the instantaneous intensities of the particle birth and death. For the case of thermal reactor, the form of under integral function was connected with the neutron generation lifetime:

$$
\lambda(s)-\mu(s)=1 /[a-b \cdot \exp (-s / c)] .
$$

The parameters $a, b$ and $c$ used in (2) are related to the parameters of breeding medium: $c=\tau_{\text {delayed }}$, where $\tau_{\text {delayed }}$ is delayed neutrons' lifetime, $b=c \cdot \beta$, where $\beta$ is the fraction of

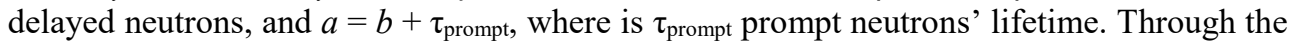
mathematical apparatus based on equation (2) the fundamental curve of neutron and breeding medium interaction was obtained (fig. 1).

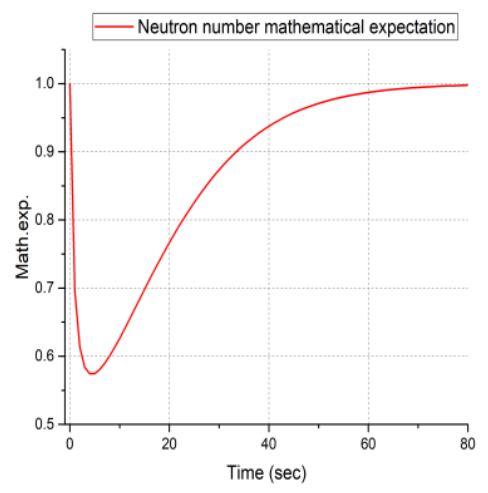

Fig. 1. The fundamental curve of neutron and breeding medium interaction.

The dependence shown in figure 1 was obtained for a simple point reactor with pure ${ }^{235} \mathrm{U}$ medium. The decline in the curve describes the process of prompt neutron "jumping" into the breeding medium and its subsequent absorption. During that absorption emitters of delayed neutrons are born. Ascending curve describes the process of this emitters decay. This fundamental curve shows the most basic ideas about the interaction of a neutron with a medium. 


\subsection{Poisson, binomial and sub-Poisson formalisms}

One of the main problem was faced is using Poisson distribution formalism. In such formalism the assumption of infinite number of nuclei is taken. From the point of view of the mathematical simplicity, this is very convenient. But for real life systems this approximation is incorrect. From the physical point of view, the application of the binomial distribution for describing nuclei decay processes inside the reactor seems more correct. But that does not allow to obtain simple analytical expressions to describe thermal pointreactor. The problem was partly solved in [5] by adding the artificial correction in function (2). But it was only showed for the fuel mass dependence from reactor operating time and had certain limitations of applicability.

As the need of some new formalism was obvious the sub-Poisson distribution was proposed. The idea was based on choosing different from under integral one (2) function with properties of Poisson and Binomial distributions. It was achieved by using function

$$
f(s)=e^{-s / c} /\left(a-b \cdot e^{-s / c}\right) .
$$

This equation (3) describes the evolution of neutron inside the breeding medium in the same way as equation (2). If the integral from (1) is solved the number of neutron generations in the system can be can be expressed like

$$
n(t)=\int_{0}^{t} f(s) d s=(c / b) \ln \left[\left(a-b \cdot e^{-t / c}\right) /(a-b)\right] .
$$

It is obvious that $n$ can only take integer values. Therefore, the time scale should be discretized as well. This discretization will give the properties of binomial distribution for current formalism without going far from Poisson. In this way the dependence between neutron generation appearance time and generation number can be defined as

$$
t(n)=c \cdot \ln \left\{\left[(b-a) e^{n \cdot b / c}\right] / b\right\} .
$$
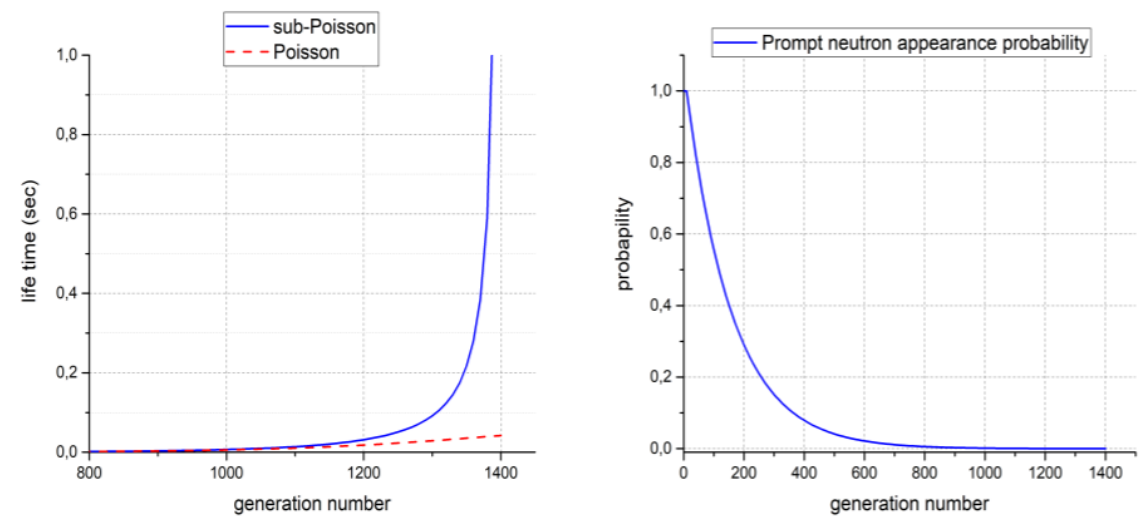

(a)

Fig. 2. The generation lifetime dependence (a) and prompt neutron appearance probability (b).

The generation lifetime now can be obtained through the difference between appearance time of two sequential generations

$$
\tau_{n}=t_{n}-t_{n-1}
$$


As was mentioned above, there is a difference between Poisson formalism from [2] and sub-Poisson formalism in current work. This difference easy to find in generation lifetime dependence on generation number (fig. 2a). As shown on a plot (fig. 2a) generation life time tends to infinity for finite generation number in case of sub-Poisson, and for infinite number for Poisson formalism. Analysis of prompt neutron appearance probability (fig. 2b) showed, that Poisson and sub-Poisson formalisms give the same dependence from generation number. These results show that the use of the current sub-Poisson formalism makes it possible to give a reasonable physical description of the emitter nuclei decay, while maintaining the convenience of analytic expressions

\section{Calculation of point-reactor reactor parameters}

To describe an operating reactor in terms of birth and death model it is necessary to find average number of neutrons on time dependence as well as neutron multiplication factor $K$. The point-reactor suites the most for this purpose as it is idealized model without any reactivity effects. For birth and death model equation (1) actually gives average number of neutrons in the medium in case of continuous timeline. To obtain the sampled time scale, we need to use equations (4-5). As was mentioned in the in the introduction the assumption of subcritical state (on prompt neutrons and with $\rho=-\beta$ ) is taken. The finite form of equation for average number was derived in details in [2] and can be used in current work

$$
M\left(t_{n}\right)=M_{0} \exp \left[\left(\rho+\beta\left(t_{n}\right)\right) \cdot t_{n} / \tau_{n}\right]
$$

The $t_{n}$ and $\tau_{n}$ in equation (7) are discrete generation appearance time and generation lifetime from equations (5-6). The $\beta\left(t_{n}\right)$ can be found through the equation

$$
\beta\left(t_{n}\right)=\beta\left(1-e^{-t_{n} / c}\right) .
$$

From the definition of neutron multiplication factor in which it equals the ratio of the neutrons average number in adjacent generations the next equation for $K_{n, n-1}$ (neutron multiplication factor between two generations) can be obtained

$$
K_{n, n-1}=M\left(t_{n}\right) / M\left(t_{n-1}\right)=\exp \left\{\left[\rho-\beta\left(t_{n}\right)\right] \cdot t_{n} / \tau_{n}-\left[\rho-\beta\left(t_{n-1}\right)\right] \cdot t_{n-1} / \tau_{n-1}\right\} .
$$

The total multiplication factor is obviously can be calculated from equation

$$
K_{t o t}=M\left(t_{n}\right) / M_{0}=\exp \left\{\left[\rho+\beta\left(t_{n}\right)\right] \cdot t_{n} / \tau_{n}\right\} .
$$

Equations (7-10) give the connection between parameters of breeding medium and operating parameters.

If $K_{t o t}$ is calculated for the last generation the case with $\tau_{n} \rightarrow \infty$ and $t_{n} \rightarrow \infty$ has place. Consequently, the asymptotic behavior of the coefficient is described by the equation

$$
K_{t o t}=\exp \left[\rho+\beta\left(t_{n}\right)\right]
$$

From this equation (11) with the corresponds to the equation (8) follows the condition of a self-sustaining reaction: $\rho=-\beta$. This result gives reason to believe that the thermal reactor is to be a safe stable system in general. 


\section{Conclusions}

The kinetics of a thermal point reactor is considered within particles birth and death model. A description of prompt and delayed neutrons behavior in the breeding medium was given. Their separation into different components made it possible to represent the reactor as a subcritical medium with an internal source of neutrons.

At the same time, the sub-Poisson formalism described in Section 2.1 with discretization of the time scale gives a reasonable physical description of the appearance process of delayed neutrons from emitter nuclei. The dependence of the lifetime of the neutron generation shown on the figure (2a) characterizes the evolution of the multiplying medium for a single specimen neutron. The equations obtained for the generation appearance time (5) and generation lifetime (6) allow us to determine the parameters of the operation of the reactor under study.

The example of a point reactor shows the derivation of the equations for the multiplication factor $(9,10)$ and the relation of the presented quantities to the reactivity $(11)$. All the expressions obtained give an integral picture of the basic neutron interaction with a breeding medium. Further work aims to scale current mathematical apparatus for different types of reactor.

\section{References}

1. D.G. Kendall, Ann. Math. Statist., v. 19, no. 1, pp. 1-15 (1948)

2. T.N. Korbut, A.V. Kuz'min, E.A. Rudak, Bulletin of the RAS. Phys., v. 79 (2015)

3. V.I. Vladimirov, Prakticheskie zadachi po ekspluatatsii yadernyih reaktorov (Practical tasks for the operation of nuclear reactors), Moscow (1986)

4. S.S. Kapoor, Pramana - journal of physics v. 59, no. 6, pp. 941-950 (2002)

5. Ed.A. Rudak, T.N. Korbut, A.V. Kuzmin, M.O. Kravchenko, «Molodezh v nauke 2.0'17» NAS of Belarus, no. 2, pp. 285-291 (2018) 